\title{
High-sensitivity temperature sensor based on anti-resonance in high-index polymer-coated optical fiber interferometers
}

\author{
Xiaokang Lian \\ Technological University Dublin, d15128010@mytudublin.ie \\ Qiang Wu \\ University of Northumbria at Newcastle \\ Gerald Farrell \\ Technological University Dublin, gerald.farrell@tudublin.ie
}

See next page for additional authors

Follow this and additional works at: https://arrow.tudublin.ie/prcart

Part of the Optics Commons

\section{Recommended Citation}

Lian X, Wu Q, Farrell G, Semenova Y. High-sensitivity temperature sensor based on anti-resonance in highindex polymer-coated optical fiber interferometers. Opt Lett. 2020 Oct 1;45(19):5385-5388. doi: 10.1364/ OL.403050. PMID: 33001900.

This Article is brought to you for free and open access by the Photonics Research Centre at ARROW@TU Dublin. It has been accepted for inclusion in Articles by an authorized administrator of ARROW@TU Dublin. For more information, please contact arrow.admin@tudublin.ie, aisling.coyne@tudublin.ie,gerard.connolly@tudublin.ie. Funder: TU Dublin

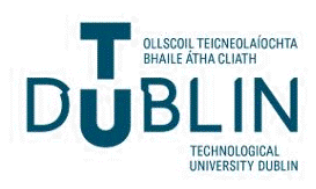


Authors

Xiaokang Lian, Qiang Wu, Gerald Farrell, and Yuliya Semenova

This article is available at ARROW@TU Dublin: https://arrow.tudublin.ie/prcart/12 


\title{
High-sensitivity temperature sensor based on anti-resonance in high-index polymer-coated optical fiber interferometers
}

\author{
Xiaokang Lian, ${ }^{1, *}$ Qiang Wu, ${ }^{2,3}$ (i) Gerald Farrell, ${ }^{1}$ and Yuliya Semenova ${ }^{1}$ \\ ${ }^{1}$ Photonics Research Centre, Technological University Dublin, Dublin D08 X622, Ireland \\ ${ }^{2}$ Department of Mathematics, Physics, and Electrical Engineering, Northumbria University, Newcastle upon Tyne NE1 8ST, UK \\ ${ }^{3}$ Key Laboratory of Nondestructive Test (Ministry of Education) of Nanchang Hangkong University, Nanchang 330063, China \\ *Corresponding author:d15128010@mytudublin.ie
}

Received 21 July 2020; revised 24 August 2020; accepted 24 August 2020; posted 25 August 2020 (Doc. ID 403050); published 21 September 2020

Compared to the multimode interference (MMI) effect, the anti-resonance (AR) effect does not rely on the multimode property of the optical waveguide. This Letter shows that fiber bending can suppress the MMI and can break the superposition of AR spectra of multiple modes in a high-index polymer-coated optical fiber interferometer based on a single-mode fiber-polymer-coated no-core fiber-single-mode fiber hetero-structure. This results in the dominance of the AR spectrum of an individual mode and consequently in periodic sharp transmission dips. As a result of this phenomenon and large thermo-optical and thermal expansion coefficients of the polymer, a compact, high-sensitivity and linear response temperature sensor with the sensitivity as high as $-3.784 \mathrm{~nm} /{ }^{\circ} \mathrm{C}$ has been demonstrated experimentally. () 2020 Optical Society of America

https://doi.org/10.1364/OL.403050

Fiber temperature sensors based on the anti-resonance (AR) effect have gained significant popularity owing to their simple structure, periodic transmission dips, high spectral extinction ratio, large free spectral range and multiplexing capability [1-3]. The simplest design for an AR-based fiber sensor is a fiber hetero-structure, such as a single-mode fiber (SMF)hollow-core fiber (HCF)-SMF (SHCS). The light guiding in the low-index air core of the HCF is based on the AR effect, which allows the anti-resonant light to be reflected back from the high-index cladding while allowing forward transmission of the resonant light [4]. The resonant coupling between the core and the cladding modes in the HCF results in periodic dips in the spectra of the SHCS [5]. The typical temperature sensitivity of a silica-based SHCS is around $30 \mathrm{pm} /{ }^{\circ} \mathrm{C}$ in the range of room temperature to over $850^{\circ} \mathrm{C}$ [1]. With a polydimethylsiloxane (PDMS) coating on the HCF, a sensitivity of $-201 \mathrm{pm} /{ }^{\circ} \mathrm{C}$ in the range from -30 to $45^{\circ} \mathrm{C}$ was achieved, owing to the large thermo-optical and thermal expansion coefficients of the polymer coating [3]. These SHCS sensors show a highly linear spectral response to temperature. The positions of the spectral dips and their sensitivity to perturbations are independent of the length of the HCF, which could facilitate the miniaturization and design of compact sensors [1-3]. However, the loss within the HCF is high, and the hollow core is prone to collapse during fusion splicing with the SMFs, which can affect the spectrum and reduce the manufacturing yield $[2,6]$.

An optical fiber interferometer for use as a temperature sensor can be based on the multimode interference (MMI) effect [7-9]. The interferometer can be a hetero-structure, such as an SMFmultimode fiber-SMF (SMS), where the multimode fiber can be replaced by a no-core fiber (NCF), small-core fiber (SCF), or any other fiber supporting multiple guided modes. The transmission spectrum of an interferometer is highly dependent on the length of the middle section fiber, where light is guided by total internal reflection. The sensitivity of temperature sensors based on a silica SMS [10], SMF-NCF-SMF (SNCS) [11], and SMF-SCF-SMF [12] is less than $40 \mathrm{pm} /{ }^{\circ} \mathrm{C}$.

In this Letter, we demonstrate a new type of temperature sensor consisting of a bent high-index polymer-coated SNCS (PC-SNCS, SMF-PC-NCF-SMF), which is based on an AR effect similar to that in an SHCS instead of the MMI effect present in an SNCS. Our previous paper [13] has shown that periodic broad dips can appear in the transmission spectra of straight PC-SNCSs with a long or bent PC-NCF (length $>50 \mathrm{~mm}$ ). We demonstrate that periodic sharp dips can be achieved for the PC-SNCSs with a millimeter order length $(<10 \mathrm{~mm})$ subjected to bending. The spectral response of the PC-SNCSs to temperature is investigated.

In this Letter, SMF28 fiber (from Corning) was used as the SMF, and FG125LA fiber (from Thorlabs) was used as the NCF. Figures 1(a) and 1(b) show the schematic diagrams of the SNCS and PC-SNCS, respectively, for simplicity sake without bending. The length of the middle section fibers is $L$. The diameter of the silica NCF is $125 \mu \mathrm{m}$. The polymer coating is the original acrylate coating as supplied by the manufacturer of the NCF and has a thickness $(t)$ of $62.5 \mu \mathrm{m}$. The refractive indices of the NCF, the polymer coating, and the surrounding air are assumed as $n_{1}=1.445, n_{2}=1.51$, and $n_{3}=1$ at a wavelength $(\lambda)$ of $1550 \mathrm{~nm}$, respectively, with $n_{2}>n_{1}>n_{3}$. 
(a)

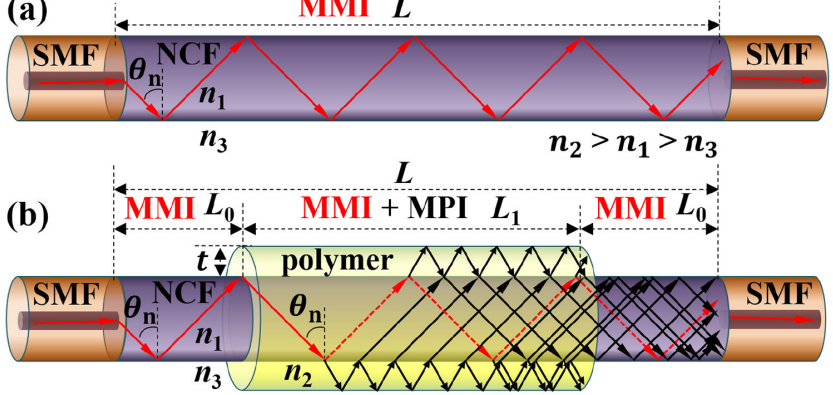

Fig. 1. Schematic diagrams: (a) SMF-NCF-SMF (SNCS) structure, (b) SMF-polymer-coated NCF-SMF (PC-SNCS) structure.

For the SNCS in Fig. 1(a), when the fundamental mode of the lead-in (left) SMF is coupled into the NCF, multiple modes $\mathrm{LP}_{0, n}$ are excited due to the on-axis alignment, which propagate through the NCF and are then coupled into the lead-out SMF. The NCF is where the MMI takes place, which determines the transmission spectrum. The ray trajectory of one $\mathrm{LP}_{0, n}$ mode, with the effective refractive index $n_{\text {eff }}=n_{1} * \sin \theta_{n}$, is indicated by the red arrows, which are confined to the NCF by total internal reflection.

In the PC-SNCS shown in Fig. 1(b), the lengths $L_{0}$ of the polymer coating $(\sim 1.5 \mathrm{~mm})$ at both ends of the PC-NCF are removed in order to enable the automatic splicing process with the SMFs. The length of the remaining polymer coating is $L_{1}$ $(<7 \mathrm{~mm})$. Using geometrical optics to illustrate propagation in the PC-NCF section, the behavior of the ray in NCF on the left side is the same as that in Fig. 1(a), as indicated by the red arrows. The ray in the PC-NCF section is partially reflected at the inner boundary of the polymer coating and totally reflected at its outer boundary, resulting in multi-path interference (MPI). The partially reflected ray indicated by the dashed red arrows retains the same angle $\left(\theta_{n}\right)$ with the radial direction as that in Fig. 1(a). The field in the straight PC-NCF section can be considered as a combined MMI and MPI. The field in the NCF on the right side is dominated by the MMI.

The MPI will cause an AR effect in the PC-NCF, and its dispersion diagram $\left(n_{\text {eff }}\right.$ versus $\lambda$ ) is different from that of the NCF [14]. Figure 2 shows the dispersion curves of the modes $\mathrm{HE}_{1, N}(N=34,35, \ldots, 38)$ in the PC-NCF and the modes $\mathrm{HE}_{1,1}\left(\mathrm{LP}_{0,1}\right)$ and $\mathrm{HE}_{1,2}\left(\mathrm{LP}_{0,2}\right)$ in the NCF, calculated using a graphical method [14]. The parts with $n_{\text {eff }}>n_{1}$ denote the cladding modes $\mathrm{HE}_{1, N}$ in the PC-NCF, showing a strong index dispersion. On the same curve, as the $\lambda$ increases, the $\mathrm{HE}_{1, N}$ will change into the anti-resonant core modes $\mathrm{HE}_{1, n+(N-n)}$, $n=1,2, \ldots$, where the subscript " $n$ " denotes the radial number in the core while the "N-n" denotes the radial number in the cladding. The $\mathrm{HE}_{1, n+(N-n)}$ modes have moderate index dispersion similar to that of the $\mathrm{HE}_{1, n}$ modes in NCF, where their dispersion curves intersect with each other. The resonant bands are indicated by the vertical black and red dashed lines, whose positions are approximated as the cutoff wavelengths of the $\mathrm{TM}_{N}$ and $\mathrm{TE}_{N}$ modes, respectively, in an equivalent asymmetric planar waveguide [14]:

$$
\lambda_{N, \mathrm{c}}=\frac{2 t \sqrt{n_{2}^{2}-n_{1}^{2}}}{\left[N-1+\frac{1}{\pi} \tan ^{-1}\left(\kappa \frac{\sqrt{n_{1}^{2}-n_{3}^{2}}}{\sqrt{n_{2}^{2}-n_{1}^{2}}}\right)\right]}, \quad \kappa=\left\{\begin{array}{cc}
1, & \mathrm{TE}_{N} \\
\frac{n_{2}^{2}}{n_{3}^{2}}, & \mathrm{TM}_{N}
\end{array},\right.
$$

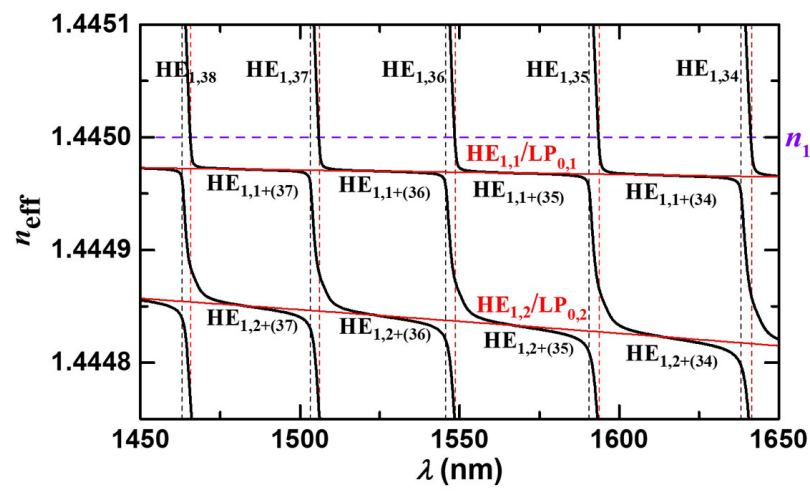

Fig. 2. (a) Dispersion curves for $\mathrm{HE}_{1,1+(N-1)}$ and $\mathrm{HE}_{1,2+(N-2)}$ $(N=34,35, \ldots, 38)$ in the PC-NCF (black lines) and $\mathrm{HE}_{1,1}\left(\mathrm{LP}_{0,1}\right)$ and $\mathrm{HE}_{1,2}\left(\mathrm{LP}_{0,2}\right)$ in NCF (red lines).

where $N=34,35, \ldots, 38$. The $n_{\text {eff }}$ of $\mathrm{HE}_{1, n+(N-n)}$ in $\mathrm{AR}$ bands can be approximated as that of $\mathrm{HE}_{1, n}\left(\mathrm{LP}_{0, n}\right)$ in NCF.

The transmission efficiency of the straight SNCS is calculated by $[7,8]$

$$
I_{\mathrm{MMI}}=10 \log _{10}\left(\left|\sum \eta_{0, n} \exp \left(i \frac{2 \pi n_{\mathrm{eff}}\left(\mathrm{LP}_{0, n}\right) L}{\lambda}\right)\right|^{2}\right),
$$

where $\eta_{0, n}$ denotes the power coupling coefficient between the fundamental mode of the SMF and the excited modes $\mathrm{LP}_{0, n}$ of the NCF. The transmission efficiency of the straight PC-SNCS due to the AR can be calculated based on an asymmetric planar reflection model described in [13]

$$
I_{\mathrm{AR}}=10 \log _{10}\left(\sum \eta_{0, n}^{2} \overline{\mathrm{R}}_{0, n}\right),
$$

where $\overline{\mathrm{R}}_{0, n}$ is the ratio of reflection intensity of a ray $\left(\mathrm{LP}_{0, n}\right)$ impinging on the asymmetric planar waveguide, which is independent of the length. The transmission efficiency of an individual $\mathrm{LP}_{0, n}$ mode can be calculated by

$$
I_{0, n}=10 \log _{10}\left(\eta_{0, n}^{2} \overline{\mathrm{R}}_{0, n}\right) .
$$

Figure 3(a) shows the transmission spectra of the individual modes $\operatorname{LP}_{0, n}(n=1,2, \ldots, 7)$ calculated by Eq. (4), which exhibit periodic dips and windows. Higher-mode orders show a red spectral shift, accompanied by a change of the depth of the dips. The intensity of the transmission windows increases at first and then reduces in accordance with the power coupling coefficient, as shown in Ref. [7]. The superposition of the spectra of individual modes $\mathrm{LP}_{0, n}$ by Eq. (3) results in a relatively flat spectrum for the straight PC-SNCS, as shown by the black curve in Fig. 3(b), where the periodic broad dips are indicated by the vertical black and red dashed lines (same as Fig. 2). The spectrum with periodic broad dips was achieved experimentally in a long PC-SNCS $(L>50 \mathrm{~mm})$ in Ref. [13].

Equations (3) and (4) consider only the AR effect; however, the actual light field in the straight PC-NCF also includes MMI as discussed. In order to better describe the situation with both AR and MMI, the beam propagation method (BPM) was employed to simulate the transmission spectra of straight PCSNCSs with $L=9 \mathrm{~mm}$ and $9.3 \mathrm{~mm}$ [9]. The results are shown in Fig. 4(a). The spectrum of the PC-SNCS with $L=9 \mathrm{~mm}$ 

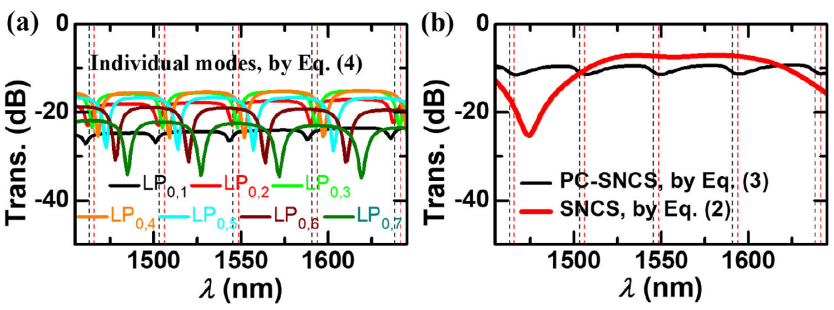

Fig. 3. Simulated transmission spectra of (a) individual modes $\mathrm{LP}_{0, n}$ calculated by Eq. (4); (b) straight PC-SNCS calculated by Eq. (3) and straight SNCS with $L=9 \mathrm{~mm}$ calculated by Eq. (2).
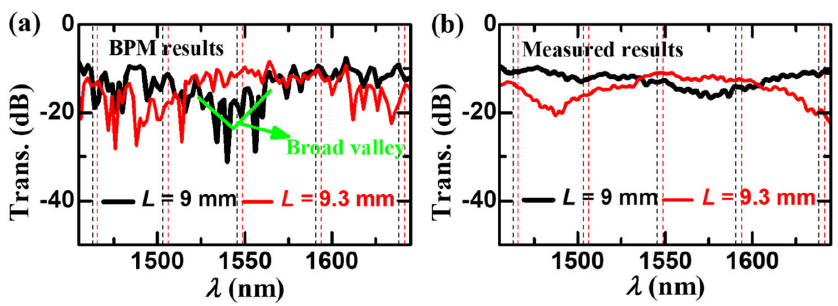

Fig. 4. (a) Transmission spectra simulated by BPM for straight PCSNCSs with $L=9 \mathrm{~mm}$ and $9.3 \mathrm{~mm}$. (b) Measured transmission spectra for straight PC-SNCSs with $L=9.0 \mathrm{~mm}$ and $9.3 \mathrm{~mm}\left(\right.$ at $\left.\sim 25^{\circ} \mathrm{C}\right)$.

shows a fringe pattern with multiple random dips and a broad valley located around $1550 \mathrm{~nm}$. The valley blueshifts towards around $1475 \mathrm{~nm}$ for the PC-SNCS with a larger $L=9.3 \mathrm{~mm}$ in Fig. 4(a). The PC-SNCS with $L=9.3 \mathrm{~mm}$ shows similar spectral features to those of the SNCS with $L=9 \mathrm{~mm}$ in Fig. 3(b), where a broad valley around $1475 \mathrm{~nm}$ is related to the destructive interference of modes in the NCF. The blue spectral shift for the PC-SNCSs with an increased $L$ in Fig. $4(\mathrm{a})$ is consistent with the trend in a MMI-based optical fiber interferometer, as shown in Ref. [8]. The simulated results are confirmed by the experiments, as shown in Fig. 4(b). Compared to the simulated results, the multiple random dips are highly suppressed in the measured spectra, which may be due to the internal defects and the surface roughness of the polymer coating [15].

The similarity of spectral characteristics of the straight PCSNCS with those for the straight SNCS and the dependence of the spectral shift on the length indicates that the MMI effect dominates in the straight PC-SNCS with a short $L$.

The resonance in PC-NCF results from the coupling between the core and the cladding modes. The resonant wavelengths depend only on the thickness and the refractive index of the high-index cladding, as indicated by Eq. (1). It can be deduced that the AR effect in PC-SNCS is less dependent on the collective behavior of the $\mathrm{LP}_{0, n}$ modes, compared to MMI. When any individual $\mathrm{LP}_{0, n}$ mode meets the coupling condition with a corresponding cladding mode, resonance will happen. The phase and the coherence of the spatial eigenmodes are sensitive to the fiber bends and twists, which induce mode coupling and the redistribution of energy among modes in multimode fibers $[16,17]$. Therefore, the bending or twisting may break the superimposed spectrum of the multiple $\mathrm{LP}_{0, n}$ modes in the straight fiber structures indicated by Eqs. (2) and (3). The AR spectrum of an $\mathrm{LP}_{0, n}$ mode owning high energy and the resonant condition may stand out, while the others are hidden and thus result in periodic sharp dips as shown in Fig. 3(a).

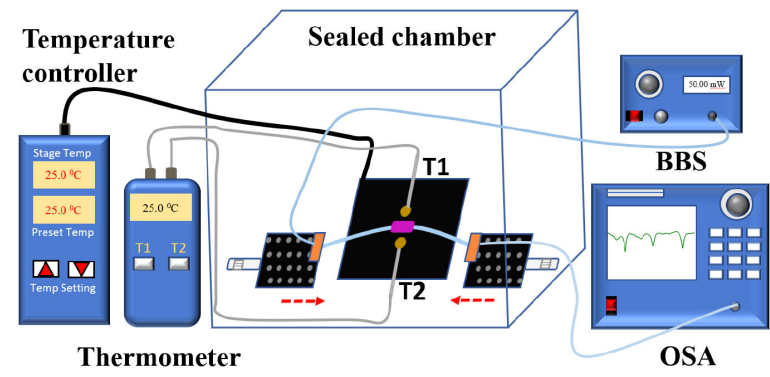

Fig. 5. Experimental setup for temperature and transmission spectrum measurement.

Figure 5 shows the experimental setup used to investigate the effect of fiber bending and temperature. The PC-SNCS fiber sample is fixed on two translation stages in contact with the surface of the temperature stage, with these three stages located in the same plane. To allow for the PC-SNCS to be bent in a controlled fashion, the bending curvature of the sample can be tuned by adjusting the distance between two translation stages. The bend radius $(R)$ was calculated based on the displacement of the translation stages [13]. After adjustment of the bending states, two K-type thermocouples $\left(T_{1}\right.$ and $\left.T_{2}\right)$ were fixed closely to the PC-NCF middle section. The measured temperature is an average of the two temperatures: $T=\left(T_{1}+T_{2}\right) / 2$. The samples and the stages were located in a sealed box, with small openings for the lead-in/lead-out wires for the temperature controller $\left(25^{\circ} \mathrm{C}-50^{\circ} \mathrm{C}\right)$, thermometer (RS 206-3738), broadband light source (BBS, Thorlabs S5FC1005s) and optical spectrum analyzer (OSA, Agilent 86142B).

Figure 6(a) shows the spectral response of the PC-SNCS with $L=9.3 \mathrm{~mm}$ under the influence of different fiber bend radii. The changes of the bend radius result in various spectral responses, corresponding to the changes of modal phases and the redistribution of energy among modes. Due to the AR nature of the PC-NCF, periodic dips are clearly observed in the spectral responses, as indicated by the vertical black and red dashed lines. The periodic dips for the PC-SNCS with $R=16.4 \mathrm{~cm}$ are broad, similar to those for the long PC-SNCS in Ref. [13]. The broad dips are related to the superpostion of the AR spectra of multiple $\mathrm{LP}_{0, n}$ modes. The spectrum for $R=10.2 \mathrm{~cm}$ has two separate dip pairs around $1470 \mathrm{~nm}, 1500 \mathrm{~nm}$ and $1550 \mathrm{~nm}$, which are expected to be related to two modes with distinct resonant wavelengths. After a slight adjustment of the bend radius, the spectrum of the PC-SNCS with $R=10 \mathrm{~cm}$ shows periodic sharp dips [redrawn in Fig. 6(b)], similar to the simulated spectra for the individual modes in Fig. 3(a). For a reduced bend radius $R=6.5 \mathrm{~cm}$, the dips become broad again, corresponding to a further change in the modal phase and energy redistribution. The experimental results indicate that the superposition of the AR spectra of multiple modes led to broadening of dips while separate individual modes caused sharper dips.

The spectra of the straight and bent $(R=10 \mathrm{~cm})$ PC-SNCS with $L=9.3 \mathrm{~mm}$ are redrawn in Fig. 6(b), with a comparison to those of the straight and bent $(R=11 \mathrm{~cm})$ PC-SNCS with $L=9 \mathrm{~mm}$ in Fig. 6(c) and those of the straight and bent $(R=15 \mathrm{~cm})$ PC-SNCS with $L=7.5 \mathrm{~mm}$ in Fig. 6(d). The results suggest that periodic sharp dips can be achieved for all the three bent PC-SNCSs with different $L$, even though their spectra for the straight fiber states are different. This result indicates 

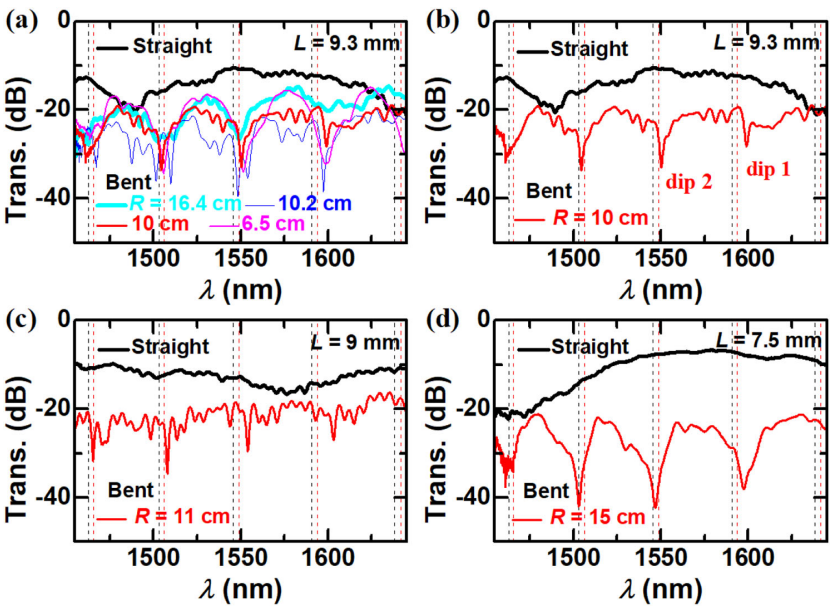

Fig. 6. Measured transmission spectra for bent and straight PC-SNCSs with (a) and (b) $L=9.3 \mathrm{~mm}$, (c) $L=9 \mathrm{~mm}$, and (d) $L=7.5 \mathrm{~mm}$ (at $\sim 25^{\circ} \mathrm{C}$, ' $R$ ' $=$ bend radius).
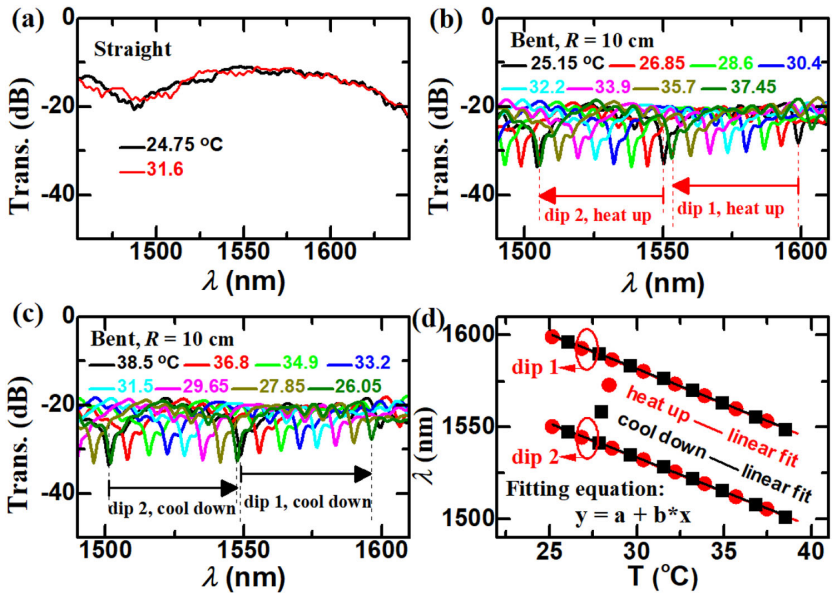

Fig. 7. Measured transmission spectra of (a) the straight, (b) and (c) the bent $(R=10 \mathrm{~cm})$ PC-SNCS with $L=9.3 \mathrm{~mm}$, at different temperatures as indicated; (d) shows the spectral shifts and the linear fittings of dip 1 and dip 2 in (b) and (c).

that for fabrication the repeatability with respect to the length of $\mathrm{PC}-\mathrm{NCF}$ is relatively robust.

The spectra of the straight PC-SNCS with $L=9.3 \mathrm{~mm}$ at $24.75^{\circ} \mathrm{C}$ and $31.6^{\circ} \mathrm{C}$ are shown in Fig. $7(\mathrm{a})$. As the temperature changes the position of the spectral valley around $1475 \mathrm{~nm}$ is almost unchanged. The results indicate that the spectral valley due to the MMI is less sensitive to temperature.

The PC-SNCS with $L=9.3 \mathrm{~mm}$ and $R=10 \mathrm{~cm}$ is employed for temperature measurement, as shown in Fig. 6(b), where the free spectral range between dip 1 and dip 2 is about $50 \mathrm{~nm}$. The measurements were taken with a step size of about $2^{\circ} \mathrm{C}$. The spectrum blueshifts within one free spectral range as the temperature increases, as shown in Fig. 7(b). As the temperature continues to increase, dip 1 will shift to the starting position (at $25.15^{\circ} \mathrm{C}$ ) of dip 2 , and the spectral shift will continue and repeat periodically. Conversely, the spectrum redshifts as the temperature decreases, as shown in Fig. 7 (c). Figure 7 (d) shows the linear fittings of the results in Figs. 7 (b) and 7 (c). The R-squared value and the sensitivity (the slope) for dip 1 in the heating process are $\mathrm{R}^{2}=0.99941$ and $-3.757 \mathrm{~nm} /{ }^{\circ} \mathrm{C}$, respectively, which are quite close to the values of $\mathrm{R}^{2}=0.99912$ and $-3.784 \mathrm{~nm} /{ }^{\circ} \mathrm{C}$ in the cooling process. The $\mathrm{R}^{2}$ and the sensitivity of the dip 2 in the heating process are 0.99890 and $-3.694 \mathrm{~nm} /{ }^{\circ} \mathrm{C}$, respectively, which are also quite close to the values of $\mathrm{R}^{2}=0.99875$ and $-3.703 \mathrm{~nm} /{ }^{\circ} \mathrm{C}$ during the cooling process. The highly linear response is similar to that observed in the AR-based SHCS [1-3]. The sensitivity achieved with the PC-SNCS in this Letter is about 19 times higher than that of the PDMS-coated SHCS reported in [3].

In conclusion, a novel type of temperature sensor based on a bent PC-SNCS has been demonstrated. The sensor has a transmission spectrum with periodic sharp dips caused by the AR effect of an individual mode, attributed to breaking of the superposition effect of the multiple guided modes induced by the fiber bending. The sensor is easy to fabricate and has a highly linear temperature response with the sensitivity of up to $-3.784 \mathrm{~nm} /{ }^{\circ} \mathrm{C}$. This Letter implies that the different degrees of dependence of the MMI and the AR effects on the multimode property can open new avenues in the design of AR-based devices, such as sensors and filters with high-index claddings.

Funding. Technological University Dublin Fiosraigh Scholarship Program 2016.

Disclosures. The authors declare no conflicts of interest.

\section{REFERENCES}

1. B. Feng, Y. Liu, and S. Qu, Opt. Eng. 55, 106127 (2016).

2. N. Cai, L. Xia, and Y. Wu, Opt. Express 26, 33501 (2018).

3. S. Liu, Y. Ji, L. Cui, W. Sun, J. Yang, and H. Li, Opt. Express 25, 18929 (2017).

4. N. M. Litchinitser, A. K. Abeeluck, C. Headley, and B. J. Eggleton, Opt. Lett. 27, 1592 (2002).

5. L. Vincetti and V. Setti, Opt. Express 18, 23133 (2010).

6. X. Zhang, H. Pan, H. Bai, M. Yan, J. Wang, C. Deng, and T. Wang, Opt. Lett. 43, 2268 (2018).

7. W. S. Mohammed, A. Mehta, and E. G. Johnson, J. Lightwave Technol. 22, 469 (2004).

8. Q. Wang, G. Farrell, and W. Yan, J. Lightwave Technol. 26, 512 (2008).

9. X. Lian, Q. Wu, G. Farrell, C. Shen, Y. Ma, and Y. Semenova, J. Lightwave Technol. 37, 1873 (2019).

10. Q. Wu, Y. Semenova, A. M. Hatta, P. Wang, and G. Farrell, Electron. Lett. 46, 1129 (2010).

11. S. Silva, E. G. P. Pachon, M. A. R. Franco, P. Jorge, J. L. Santos, F. X. Malcata, C. M. B. Cordeiro, and O. Frazão, J. Lightwave Technol. 30, 3569 (2012).

12. J. Wu, Y. Miao, B. Song, W. Lin, K. Zhang, H. Zhang, B. Liu, and J. Yao, Opt. Commun. 340, 136 (2015).

13. X. Lian, G. Farrell, Q. Wu, W. Han, C. Shen, Y. Ma, and Y. Semenova, "Spectral dependence of transmission losses in high-index polymer coated no-core fibers," J. Lightwave Technol. (to be published).

14. X. Lian, G. Farrell, Q. Wu, W. Han, C. Shen, Y. Ma, and Y. Semenova, Opt. Express 28, 16526 (2020).

15. C. C. Katsidis and D. I. Siapkas, Appl. Opt. 41, 3978 (2002).

16. D. Marcuse, Theory of Dielectric Optical Waveguides, 2 nd ed. (Academic, 1991).

17. J. M. Kahn, K. Ho, and M. Shemirani, in Optical Fiber Communication Conference (OFC) (2012), paper OW3D.3. 